'Departamento de Ginecología y Obstetricia, Hospital General Regional número uno, Instituto Mexicano del Seguro Social, Ciudad Obregón. Sonora, México.

2Departamento de Pediatría, Hospital General Regional número uno, Instituto Mexicano del Seguro Social, Ciudad Obregón. Sonora, México.

Los autores declaran no tener conflictos de interés.

El presente trabajo no recibió financiamiento externo.

Recibido el 14 febrero de 2019, aceptado el 22 de abril de 2019.

Correspondencia a: Jaime Guadalupe Valle Leal Río Juncar 1303 Fraccionamiento Puente Real, sección Miraflores, Ciudad Obregón. Sonora, México. vallejaime1@hotmail.com

\section{Adherencia terapéutica y control glucémico en pacientes con diabetes gestacional bajo dos esquemas de tratamiento}

\author{
LINET CHÁVEZ GARCÍA ${ }^{1}$, JAIME GUADALUPE VALLE LEAL², \\ CINDY JIMÉNEZ MAPULA ${ }^{1}$, SAMANTHA MELISSA \\ QUINTERO MEDRANO ${ }^{1}$, MIRIAM NAYELI LÓPEZ VILLEGAS ${ }^{1}$
}

\section{Gestational diabetes adherence to treatment and metabolic control}

Background: Adherence to treatment is a large obstacle in the management of chronic diseases. Aim: To evaluate therapeutic adherence and its relationship with glycemic control in patients with gestational diabetes using two types of treatment. Material and Methods: The Measurement of Treatment Adherence (MAT) questionnaire was applied to 93 patients with gestational diabetes. Fifty-two used metformin 41 were treated with insulin. Obstetric and socio-demographic data were collected. Results: A higher therapeutic adherence was associated with a better glycemic control. Women with a higher education level had a better adherence to treatment. The adherence and metabolic control were higher in women treated with metformin. Conclusions: Therapeutic adherence is an important factor for adequate glycemic control.

(Rev Med Chile 2019; 147: 574-578)

Key words: Hyperglycemia; Insulin; Metformin; Pregnancy; Treatment Adherence and Compliance.
$\mathrm{E}^{\mathrm{n}}$ n México, la prevalencia de diabetes gestacional (DG) se reporta entre 8,7 a $17,7 \%$. Las mexicanas están en mayor posibilidad de desarrollar DG por pertenecer a un grupo étnico de alto riesgo ${ }^{1}$.

El tratamiento de la DG está diseñado para reducir la morbilidad perinatal ${ }^{2}$, este incluye: modificaciones al estilo de vida, ejercicio de intensidad leve a moderada, cambios en alimentación y tratamiento farmacológico con insulina o hipoglucemiantes orales ${ }^{3-5}$.

La insulina no atraviesa la barrera placentaria, sus desventajas incluyen la necesidad de inyecciones, riesgo de hipoglucemia, aumento del apetito y de peso. Entre los hipoglucemiantes orales, la Metformina es el más utilizado, atraviesa la barrera placentaria, no se asocia con anomalías fetales si se usa durante el primer trimestre del embarazo y su administración es segura en el segundo y tercer trimestre de la gestación ${ }^{6,7}$.

La probabilidad de complicaciones materno-fetales tiene relación con la concentración de glucosa plasmática materna, este riesgo se reduce manteniendo adecuado control glucémico en el primer trimestre del embarazo ${ }^{8}$.

La adherencia terapéutica, definida por la Organización Mundial de la Salud (OMS) como el grado en que el comportamiento de una persona se compromete con las recomendaciones acordadas de un prestador de asistencia sanitaria, mejora el control glicémico, disminuye el riesgo de complicaciones y puede disminuir la mortalidad del producto hasta 15 veces $^{1,9,10}$.

El objetivo de estudio fue determinar que la 
falta de adherencia terapéutica está asociada a un menor control glucémico en un grupo de pacientes con DG atendidas en un hospital de segundo nivel de atención en el Noroeste de México.

\section{Material y Métodos}

Previa autorización del Comité Local de Investigación y Ética de la investigación en Salud, se realizó un estudio transversal, en mujeres gestantes con diagnóstico de diabetes gestacional, diagnostico establecido de acuerdo a los criterios de la Guía de diagnóstico y tratamiento de diabetes gestacional ${ }^{11}$, (glucosa plasmática en ayunas entre 100 y $125 \mathrm{mg}$ / $\mathrm{dl}$ valor repetido en dos determinaciones en el curso de la misma semana; y/o glucosa plasmática a las $2 \mathrm{~h}$ pos estímulo con $75 \mathrm{~g}$ de glucosa anhidra $\geq \mathrm{a}$ $140 \mathrm{mg} / \mathrm{dl}$ ); en tratamiento con insulina o metformina, y que se atendieron en consulta de obstetricia, perinatología o tococirugía de un hospital de segundo nivel de atención del noreste de México entre noviembre 2017 a enero 2018. El muestreo se realizó por casos consecutivos, excluyendo a aquellas pacientes que utilizarón fármacos esteroideos durante los últimos 3 meses, previos al estudio.

El tamaño de la muestra se calculó utilizando una fórmula para estimar y determinar dos proporciones, con un nivel de confianza de 95\%, con un poder estadístico de $80 \%$ y un nivel de significancia de $0,05 \%$.

Se establecieron 2 grupos de pacientes, tomando en cuenta el tratamiento utilizado. El grupo uno, integrado por las pacientes bajo tratamiento con metformina, el grupo 2 integrado por pacientes bajo tratamiento con insulina. En las pacientes de los dos grupos se realizaron las siguientes mediciones: glucosa $2 \mathrm{~h}$ postprandial (pos desayuno), a las 2-4 semanas posterior al inicio del tratamiento. Para determinar control no se midió hemoglobina glucosilada, ya que esta prueba no se realiza de rutina en pacientes con DG, además de ser considerada por la ALAD $2016^{11}$ como una herramienta de poco valor para establecer control en DG.

Todas las pacientes que participaron otorgaron su consentimiento por escrito y posteriormente llenaron un cuestionario con variables sociodemográficas (edad, escolaridad, residencia, ocupación y estado civil). Se interrogó sobre el antecedente o no de diabetes gestacional en embarazos previos. Las mediciones de glucosa $2 \mathrm{~h}$ postprandial se hicieron a través del sistema de análisis automatizado del equipo Unicel DXC 600, Beckman Coulter $^{12}$, se consideró control glicémico a toda paciente que presentó glucosa $2 \mathrm{hrs}$ postprandial $\leq 120 \mathrm{mg} / \mathrm{dL}$ según lo establecido por la guía de práctica clínica para diagnóstico y tratamiento de la diabetes en el embarazo ${ }^{1}$ y $\mathrm{ALAD}^{11}$, actualizaciones 2016.

Los datos referentes a adherencia terapéutica fueron recolectados por medio del cuestionario Medida de Adhesión a los Tratamientos (MAT). Este instrumento fue adaptado y validado en Portugal, con buena consistencia interna en la condición de respuesta en la forma de escala de Likert (coeficiente alfa de Cronbach de 0,74) adecuado para el portugués de Brasil en 2001, enfocada hacia Metformina e insulina, traducida $y$ adecuada al español por Gomes y colaboradores en 2014, y aplicada a adultos con diabetes mellitus tipo 2 (DM2). El cuestionario fué aplicado en este estudio a pacientes con diabetes gestacional ya que no se cuenta en la literatura con uno enfocado específicamente a diabetes gestacional. El Instrumento consta de 7 ítems con 6 posibles respuestas, con resultados que van del "siempre" al "nunca" a los que se otorga valores del 1 al 6. La adhesión fue determinada por el promedio global del instrumento, es decir, se sumaron los puntajes de cada ítem y se dividieron por el número de ítems (siete). Valores de 6 a 5 indican adhesión alta al tratamiento, de 3 a 4 adhesión media y de 1 a 2 adhesión baja ${ }^{9,12}$.

Los resultados se registraron en una base de datos en el programa Excel y fueron procesados en Software SPSS versión 25. Se determinó frecuencia de adherencia terapéutica y control glucémico en la población estudiada y se determinó la asociación entre adherencia terapéutica con control glucémico, escolaridad, estado civil, ocupación y antecedente de diabetes en embarazos previos, para ambos grupos. Para variables cualitativas utilizamos porcentajes, para variables cuantitativas fue mínima, máxima, media y desviación estándar (DE). Se estableció significancia estadística cuando el resultado de $\mathrm{p}$, obtenido por prueba exacta de Fisher y $\chi^{2}$ de Pearson fue menor a 0,05.

\section{Resultados}

Se estudiaron un total de 93 pacientes con DG, 52 bajo tratamiento con metformina (66\%), 41 
Tabla 1. Diferencia de medias de variables de pacientes con diagnóstico de Diabetes Mellitus gestacional. $\mathbf{N}=93$

\begin{tabular}{|lccc|}
\hline Variable & $\begin{array}{c}\text { Insulina } \\
\text { Media (DE) }\end{array}$ & $\begin{array}{c}\text { Metformina } \\
\text { Media (DE) }\end{array}$ & p \\
\hline Glucosa 2 h postprandial & $126,05(19,08)$ & $110,89(17,58)$ & 0,000 \\
Semanas de gestación al Dx & $20,93(6,7)$ & $24,06(5,5)$ & 0,016 \\
\hline Edad & $29,10(5,39)$ & $27,81(4,82)$ & 0,228 \\
\hline
\end{tabular}

$\mathrm{P}=\mathrm{t}$ de Student; * = significancia estadística; $\mathrm{h}=$ horas; $\mathrm{Dx}=$ diagnóstico; $\mathrm{DE}=$ desviación estándar.

con insulina (44\%). Para el grupo metformina se encontró una media de edad de 27,8 años (DE 4,82 ), para el grupo insulina una media de 29,1 años (DE 5,39). Las semanas de edad gestacional al diagnóstico fue mayor para el grupo de metformina, con una media de 24,05 (DE 5,5) semanas de edad gestacional. Las cifras mediciones de

\section{Tabla 2. Asociación de variables con adherencia terapéutica en pacientes en tratamiento para Diabetes Mellitus gestacional. $\mathbf{N}=\mathbf{9 3}$}

\begin{tabular}{|c|c|c|c|}
\hline & \multicolumn{3}{|c|}{ Adherencia terapeutica } \\
\hline & $\begin{array}{c}\text { Alta } \\
\text { n }(\%)\end{array}$ & $\begin{array}{l}\text { Media } \\
\text { n (\%) }\end{array}$ & $\mathbf{p}$ \\
\hline $\begin{array}{l}\text { Edad } \\
18 \text { a } 26 \text { años } \\
>\text { de } 26 \text { años }\end{array}$ & $\begin{array}{l}26(84) \\
49(80)\end{array}$ & $\begin{array}{r}5(16) \\
12(20)\end{array}$ & 0,679 \\
\hline $\begin{array}{l}\text { Ocupación } \\
\text { Ama de casa } \\
\text { Trabajadora }\end{array}$ & $\begin{array}{l}42(82) \\
34(81)\end{array}$ & $\begin{array}{l}9(18) \\
8(19)\end{array}$ & 0,862 \\
\hline $\begin{array}{l}\text { Escolaridad } \\
\text { Sec y Bachiller } \\
\text { Lic y Post }\end{array}$ & $\begin{array}{l}56(77) \\
20(95)\end{array}$ & $\begin{array}{c}16(23) \\
3 \quad(5)\end{array}$ & 0,069 \\
\hline $\begin{array}{l}\text { Medicamento } \\
\text { Insulina } \\
\text { Metformina }\end{array}$ & $\begin{array}{l}29(71) \\
47(90)\end{array}$ & $\begin{array}{r}12(29) \\
5(10)\end{array}$ & $0,015^{*}$ \\
\hline $\begin{array}{l}\text { Control glucémico } \\
\text { No (> } 120 \mathrm{mg} / \mathrm{dl}) \\
\text { Sí ( } \leq 120 \mathrm{mg} / \mathrm{dl})\end{array}$ & $\begin{array}{l}17(22) \\
59(78)\end{array}$ & $\begin{array}{r}16(94) \\
1(6)\end{array}$ & $0,000^{*}$ \\
\hline
\end{tabular}

ase tomó como control glicémico a todas aquellas pacientes que tuvieron igual o debajo de $120 \mathrm{mg} / \mathrm{dl}$ en muestra de sangre central a las $2 \mathrm{~h}$ postprandial, según lo establecido por la Guía de Práctica Clínica para el diagnóstico y tratamiento de la diabetes en el embarazo, última actualización 2016. $\mathrm{p}=$ prueba exacta de Fisher; $\%=$ porcentaje; $>=$ mayor $\mathrm{a}_{;} \leq=$menor o igual $\mathrm{a} ;{ }^{*}=$ Significancia estadística; $\mathrm{Sec}=$ educación secundaria; Lic $=$ Licenciatura; Post $=$ Postgrado. glucemia (glucosa $2 \mathrm{~h}$ postprandial) fueron mayores en el grupo de insulina, con una media de 126,05 mg/dl (DE 19,08), comparado con 110,89 $\mathrm{mg} / \mathrm{dl}(\mathrm{DE} 17,58)$ en el grupo de Metformina, $\mathrm{p}$ de 0,000 (Tabla 1 ).

Respecto a adherencia al tratamiento, 90\% del grupo de metformina tenía alta adherencia al tratamiento, comparando con $71 \%$ en el grupo de insulina, $p$ de 0,015 . Las pacientes con estudios de licenciatura y postgrado presentaban una mayor proporción de adherencia alta al tratamiento (95\%). El 78\% de pacientes con alta adherencia terapéutica presentaban control metabólico con cifras de glucosa $2 \mathrm{~h}$ postprandial igual o menor a $120 \mathrm{mg} / \mathrm{dl}$, comparado con el grupo con mediana adherencia en la cual solo $6 \%$ presentaba control metabólico, p de 0,000 (Tabla 2).

El control metabólico se logro en $51 \%$ de pacientes con insulina y en $69 \%$ de pacientes con metformina, $\mathrm{p}$ de 0,285 . El control metabólico se logro en $78 \%$ de los pacientes con alta adherencia al tratamiento, contra solo $6 \%$ en el grupo de mediana adherencia, p 0,000 (Tabla 3).

Tabla 3. Asociación de variables con control metabólico en pacientes en tratamiento para Diabetes Mellitus gestacional. N $=93$

\begin{tabular}{|c|c|c|c|}
\hline & \multicolumn{2}{|c|}{ Control metabólico } & \multirow[b]{2}{*}{$\mathbf{p}$} \\
\hline & $\begin{array}{c}\text { No } \\
n \quad(\%)\end{array}$ & \begin{tabular}{c}
\multicolumn{2}{c}{ Sí } \\
n
\end{tabular} & \\
\hline \multicolumn{4}{|l|}{ Medicamento } \\
\hline Insulina & $17(41)$ & $24(59)$ & \multirow[b]{2}{*}{0,285} \\
\hline Metformina & $16(31)$ & $36(69)$ & \\
\hline \multicolumn{4}{|c|}{ Adherencia terapéutica } \\
\hline Alta & $17(22)$ & $59(78)$ & \multirow{2}{*}{$0,000^{\varepsilon^{*}}$} \\
\hline Media & $16(94)$ & $1(6)$ & \\
\hline
\end{tabular}

$\&=$ prueba exacta de Fisher, $\%=$ porcentaje; * = Significancia estadística; $\mathrm{n}=$ número. 


\section{Discusión}

En este estudio se encontró que la adecuada adherencia terapéutica está relacionada al control glicémico en pacientes con DG (P de 0,000), sin embargo, se encontraron 17 (22\%) pacientes que a pesar de tener adherencia alta al tratamiento, no presentaron control glicémico, esto pudiera deberse a variables no estudiadas durante esta investigación.

Nuestros resultados concuerdan con lo expresado por la guía para el diagnóstico y tratamiento de la diabetes en el embarazo ${ }^{1}$, la American Diabetes Association ${ }^{6}$ y la OMS en Adherence to long-term therapies ${ }^{10}$, quienes refieren que, a mayor adherencia terapéutica, mejor control glicémico, sin especificar relación con el tipo de medicamento utilizado.

Concordamos con lo reportado por Díaz-Romero $^{5}$ con respecto al no control a pesar de la adherencia quien expresa, que medir la adherencia terapéutica es un indicador útil para el médico y personal encargado del control prenatal de las diabéticas durante su embarazo. Gomes-Villas ${ }^{9}$, encontró mayor adherencia a insulina que a hipoglucemiantes orales con la aplicación de la medida de adherencia al tratamiento utilizada en este estudio, pero con una población de 90 adultos con diagnóstico de DM2. Estos resultados son distintos a los de este estudio, donde encontramos una adherencia alta en $90 \%$ en pacientes del grupo de metformina, comparado con $71 \%$ en el grupo de insulina.

En lo que respecta a las características sociodemográficas, se encontró adherencia alta al tratamiento en pacientes con estudios de licenciatura y postgrado, esto se puede relacionar con la mayor capacidad para seguir instrucciones, y entender las complicaciones de no llevar un adecuado manejo terapéutico, en las pacientes con mayor escolaridad. La edad, ocupación no se asociaron al nivel de adherencia terapéutica.

Se reconocen las limitaciones del estudio, ya que la diabetes gestacional es una enfermedad compleja, multisistémica, en la que interfieren otros factores que no pudieron ser medidos en el presente estudio, y que probablemente interfieran en el control glicémico, como pueden ser con respecto al tipo de medicamento utilizado, para metformina, la dosis del medicamento, la necesidad de agregar insulina o cambiar por completo la terapia a insulina solamente, la fecha de inicio de la terapia, la dieta, pudiendo ser esta no adecuada. En cuanto a la insulina, las unidades de insulina utilizadas, los intervalos de dosificación de la misma establecidos por el médico tratante, el tipo de insulina utilizada, la fecha de inicio del tratamiento y, la dieta no adecuada. Otra limitante puede ser el sesgo de información ya que no podemos estar seguros que las pacientes contesten verazmente las preguntas de la MAT.

Por lo anterior, podemos considerar a la adherencia terapéutica como un importante factor para el adecuado control glucémico en la población estudiada, abriendo con ello un área de oportunidad para aumentar el número de pacientes que logran el control glucemico.

Es importante al momento de realizar el diagnóstico e iniciar un tratamiento farmacológico, explicar a detalle al paciente los beneficios del adecuado control glucémico y de la adecuada adherencia al tratamiento para su salud y la del producto que gesta. Para ello es importante lograr una comunicación efectiva con la paciente, en un lenguaje claro y detallado; es necesario un seguimiento cercano que permita evaluar la respuesta al manejo y poder facilitar al binomio condiciones optimas de salud.

\section{Conclusión}

La adecuada adherencia terapéutica está asociada al control glucemico en pacientes con Diabetes Gestacional bajo tratamiento con insulina y metformina adscritos a los servicios del departamento de Ginecología y Obstetricia de nuestra unidad hospitalaria. Se identifico una mayor adherencia al tratamiento en pacientes con escolaridad alta (licenciatura y postgrado). La adherencia terapéutica y el control metabólico fué mayor en el grupo de Metformina. Es importante realizar capacitaciones a las pacientes con diagnóstico de DG y hacer énfasis en la adecuada adherencia al tratamiento establecido por el personal de salud, para así disminuir complicaciones materno-fetales.

Agradecimientos: Los autores expresan su agradecimiento a personal del departamento de Ginecología y Obstetricia, personal de la coordinación de educación e investigación en salud, 
y personal de laboratorio clínico del Hospital General Regional 1 de Ciudad Obregón, Sonora, por las facilidades para la realización del estudio.

\section{Referencias}

1. IMSS. Instituto Mexicano del Seguro Social. Diagnóstico y tratamiento de la diabetes en el embarazo 2016. Disponible en: www.imss.gob.mx/sites/all/statics/guiasclinicas/320GER.pdf [Consultado el 15 de agosto de 2017].

2. Buitrago-Leal M, Molina-Giraldo S. Seguridad y efectividad de los hipoglucemiantes orales en mujeres con diabetes gestacional. Ginecol Obstet Mex 2014; 82 (7): 454-64. Disponible en: www.medigraphic.com/pdfs/ ginobsmex/gom-2014/gom147d.pdf [Consultado el 15 de agosto de 2017].

3. Buchanan T, Xiang AH, Page K. Gestational Diabetes Mellitus: Risks and Management during and after Pregnancy. Nat Rev Endocrinol 2015; 8 (11). Disponible en: www.ncbi.nlm.nih.gov/pmc/articles/PMC4404707/pdf/ nihms438633.pdf [Consultado el 9 de julio de 2017].

4. Salat D, Aguilera C. Tratamiento actual de la diabetes gestacional. Preguntas y respuestas en farmacología clínica. Med Clin Barc 2015; 145 (6): 269-72. Disponible en: https://www.icf.uab.cat/assets/pdf/productes/preres/ preres55.pdf [Consultado el 9 de julio de 2017].

5. Díaz-Romero RM, Mendoza-Flores ME, Belmont-Padilla J. Validación de un instrumento para evaluar la adherencia terapéutica en diabéticas durante el embarazo. Perinatol Reprod Hum 2004; 18 (4): 217-24. Disponible en: www.inper.mx/descargas/pdf/validaciondeunInstrumentoparaEvaluar.pdf [Consultado el 18 de agosto de 2017].

6. American diabetes association. Standards of medical care in diabetesd 2017: summary of revisions. Diabetes Care 2017; 40 (1): S4-S5. Disponible en: http://care. diabetesjournals.org/content/diacare/40/Supplement_1/ S4.full.pdf [Consultado el 14 de junio de 2017].

7. Zhao LP, Sheng XY, Zhou S, Yang T, Ma LY, Zhou Y, et al. Metformin versus insulin for gestational diabetes mellitus: a meta-analysis. Br J Clin Pharmacol 2015; 80 (5): 1224-34. Disponible en: www.ncbi.nlm.nih.gov/ pmc/articles/PMC4631195/pdf/bcp0080-1224.pdf.

8. Scottish Intercollegiate Guidelines Network. Management of Diabetes: A national clinical guidelines. 2013. Disponible en: www.sign.ac.uk/assets/sign116.pdf [Consultado el 15 de agosto de 2017].

9. World Health Organization. Adherence to long-term therapies: Evidence for action. 2003. Disponible en: www.who.int/chp/knowledge/publications/adherence_full_report.pdf?ua $=1$ [Consultado el 03 de agosto de 2017].

10. Gomes-Villas Boas LC, Lima MLSAP, Pace AE. Adhesión al tratamiento de la diabetes mellitus: validación de instrumentos para antidiabéticos orales y insulina. Rev Latino-Am Enfermagem 2014; 22 (1). Disponible en: www.scielo.br/pdf/rlae/v22n1/es_0104-1169rlae-22-01-00011.pdf [Consultado el 02 de agosto de 2017].

11. Salzberg S, Alvariñas J, López G, Gorbán de Lapertosa S, Linari MA, Falcón E, et al. Guías de diagnóstico y tratamiento de diabetes gestacional: ALAD 2016. Rev ALAD 2016; 6: 155-69. Disponible en: www. alad-americalatina.org/wp-content/uploads/2016/12/ Guias_ALAD_DG_2016.pdf [Consultado el 22 de julio de 2017].

12. Servonenet A, Thefenne H, Boukhira A, Vest P, Renard C. Evaluation of the Unicel DXC 600 (Beckman Coulter) and comparison whit integra 800 (Roche Diagnostic). Ann Biol Clin 2007; 65 (5): 555-62. Disponible en: www.jle.com/download/abc-275774 evaluation_des_performances_analytiques_du_systeme_unicel_tm_dxc_600_beckman_coulter_et_etude_de_la_ transfer--WmYtM38AAQEAAHOTsUsAAAAG-a.pdf [Consultado el 14 de agosto de 2015].

13. Barata-Delgado A, Lima ML. Contributo para validação concorrente de uma medida de adesão aos tratamentos. Psicol Saúde Doenças 2014; 2 (2): 81-100. Disponible en: www.scielo.mec.pt/pdf/psd/v2n2/v2n2a06.pdf [Consultado el 3 de agosto de 2017]. 